P. Rohland: Die Klärung and Reinigung der städtischen Abwässer. (Zeitschr. öffentI. Chem. 1914, 20, 415-419.)

P. Rohland: Das Kolloidtonreinigungsverfabren für die Abwässer der Brauereien etc. II. (Zeitschr. f, d. ges. Brauwesen 1914, 87, 413-416.)

\title{
Geheimmittel, Spezialitäten etc.
}

Armin Röhig: Geheimmittel. (Jahresbericht der chemischen Untersuchungsanstalt Leipzig 1914,49-53.) - Blanca von J. Heller, Leipzig-Möckern, Mittel gegen Nasenröte. Preis $3 \mathrm{Mk} .23 \mathrm{ccm}$ einer farblosen, sauren Flüssigkeit mit mineralischem Bodensatz, eine Auflosung von 1,44\% Kupferchlorid in $0,8 \%$ Salzsäure, versetzt mit $4,5 \%$ Speckstein. - Wundsalbe von Apotheker Grissinger, Ründeroth (Rhld,), Preis $1 \mathrm{Mk}$. Ist ein Mineralfett mit 5,6\% Zinkoxyd. E. Bark's schmerzstillende Einreibung gegen Rheumatismus, HexenschuB usw. Preis $0,75 \mathrm{Mk} .48 \mathrm{com}$ einer trüben, grünlichen Flüssigkeit mit zwei Schichten; die obere Schicht besteht aus Terpentinöl und Ammoniak, die untere Schicht enthält Lorbeeröl. - Brom-Somnisan, Tutogen-Laboratorium Szittkehmen. Gegen nervöse Störungen aller Art und Schlallosigkeit. Preis 2,50 Mk. $140 \mathrm{ccm}$ einer kaffeebraunen, nach Baldrian riechenden Flüssigkeit mit 50,2 Vol. $\%$ Alkohol, 17,16\% Extrakt, ist eine Mischung von Somnisan (Baldrianauzzug nach Art der Fluidexirakte) mit $5 \%$ einer Bromsalzmischung. - Colso von Apotheker Dr. A. Uecker, Niewerle, N.-Lausitz. Mittel gegen Trunksucht. Preis 5,50 Mk. Flasche mit $252 \mathrm{ccm}$ einer trüben, schwach kaffeebraunen Flüssigkeit, darin nachgewiesen 6,24 Vol.- $\%$ Alkohol, 14,42\% Extralst, ein Pflanzenextrakt mit Zusatz von Zucker und Glycerin. - Enokturin-Tabletten des Institutes Sanis, München, ein Nähr- und Kräftigungsmittel ersten Ranges; Befreiung von Bettnässen. Preis $11 \mathrm{Mk}$. Zu einer Kur gehören 6 Schachteln. $42 \mathrm{Ta}-$ bletten zu je $1,03 \mathrm{~g}$ einer Mischung von 80 Teilen Zucker mit 20 Teilen eines Nährpräparates nach Art des Sanatogen oder Biocitin, Verkaufswert etwa 25 Pfg. - Enos* Tabletten, Apotheke Burgbernheim, gegen Bettnässen. Preis 3.50 Mk. 47 linsenförmige Tabletten aus Getreidestärke, Kakao und Glycerin; der angegebene Gehalt an Castoreum (Bibergeil) war ebensowenig festzustellen als die bekannten spezifischen Bettnäßmittel. - Thyriotin der Gesellschaft Thyriota Comp., Hanau, Mittel gegen Haarausfall und Kahlköpfigkeit; Preis der Flasche 3,50 Mk. $130 \mathrm{ccm}$ einer grünlichgelben Flüssigkeit mit 94,69 Vol. $\%$ Alkohol, mit Ammoniak alkalisch gemacht. - Matein, Ulrich Hobl's, mit Broschüre über "Energieschwund der Nervositäts. Grund"; gibt neue Kraft. Preis $9 \mathrm{Mk} .200$ Pastillen, je $0,5 \mathrm{~g}$, grauweib, süß schmeckend, mit Pflaumenaroma, enthalt Coffein und Zucker; ist wabrscheinlich eine Zubereitung aus Paraguaytee, Blättern von llex-Arten, mit Zucker und Stärke. Boranium-Beeren der Dearborn, Ges. m. b. H., Berlin. Konfekt zur Herabminderung der Fettleibigkeit. Preis 7,50 Mk. 126 halbkugelförmige Bonbons im Gewicht von $0,85-2,10 \mathrm{~g}$, Geschmack nach Pfefferminzöl, künstlich gefärbt; enthalten als Arzneistoff rund $0,04 \mathrm{~g}$ Phenolphthalein in einem mittelgroben Stück, sie gehören daher zu den Laxinkonfekten, die von der Ministerialverordnung vom 31 . Juli 1913 betroffen werden. - Eksip von W. Richartz, Bonn a. Rh., Antidiabeticum. Preis $12 \mathrm{Mk}$. für die Kur. Flasche mit $120 \mathrm{ccm}$ eines dünnen wässerigen gerbstoffhaltigen Pflanzenauszuges mit Zusatz von $6 \%$ offizineller Salzsäure. - Thermopod von Dr. C. Schweitzer, Heilbronn, Mittel gegen kalte Füße. Preis $1 \mathrm{Mk}$; eine Zinntube mit einer rötlichgelben Salbe, einer Verreibung von Vaseline, Serföl und Paprikaauszug, aromatisiert mit Amylacetat. - Citrophen-Tabletten gegen Kopfschmerz, Influenza usw., wirkt schlaf́bringend, appetitanregend. Preis $1 \mathrm{Mk}$. Schachtel mit 28 Stück Tabletten zu $0,5 \mathrm{~g}$, aus einem zuckerhaltigen Mantel und einem Kern bestehend; letzterer enthält nachweisbar Monophenetidincitrat als wirksamen Stoff. - Regulaxier von L. Graf, 
Leipzig, Lößniger Str. 6, 4 Tr. r., Abführmittel, im Hausierhandel entnommen. Schachtel mit 20 runden Tabletten zu $0,55 \mathrm{~g}$, Geschmack nach Vanillin, aus Zucker und Phenolphthalein je $0,05 \%$ pro Tablette. (Vergl. Ministerialverordnung vom 31. Juli 1913.)

- Dellheim's Brust- and Blutreinigungstee von A. Dellheim, Weinheim. Paket 1 Mk., mit 95 g einer Teemischung, darin Rhizom. Gramin., Herba Equiseti, Herba Urticae, Flores Malvae, Herb. Asperulae und Rad. Sarsaparill. - Bisurierte Magnesia der To Kalon Manufacturing Co. Lid. London W, ,eine sauere neutralisierte Komposition zur Verbütung von Verdauungsstörungen". Preis 2,50 Mk. Schachtel mit $40 \mathrm{~g}$ eines weißen, schwach alkalisch schmeckenden Pulvers, bestehend aus Natrium bicarb., Magnesium carbon. und basisch. Wismutcarbonat. - Nervenmark Trabol von Apotheker Gronwald \& Co., Berlin SW. „Von guter Wirkung bei allen Schwächezuständen, schafft stahlharte Nerven und eine kräftige Muskulatur"s. Preis $3 \mathrm{Mk}$. Papierbeutel mit $120 \mathrm{~g}$ eines gelblichen, nach Biskuit schmeckenden Pulvers, Hauptbestandteil Trockenmilch und Lecithin nach Art des Biocitins. Angeblich soll noch Hämoglobin und Apfelsäure zugesetzt sein. - Rheumatermin von Apotheker Gronwald \& Co., Berlin SW., „ruft aufgetragen, wohliges Behagen hervor, Schmerzen verlieren sich schon nach der ersten Auflage". Preis $1,65 \mathrm{Mk}$. Zinntube mit $62 \mathrm{~g}$ einer gelblich-weißen nach Koniferenöl riechenden Salbe, bestehend aus Wollfett mit Zusatz von rund $5 \%$ einer Salzmischung nach Art des Wiesbadener Kochbrunnensalzes. - Salrado Compound von The Salrado Company London 68, Schönheits- und Kräftigungsmittel. Preis $2 \mathrm{Mk}$. Flasebe mit $50 \mathrm{cem}$ einer kaffeebraunen, schwach alkalischen, wässerigen Flüssigkeit, in der sicher nachweisbar war ein Pflanzenextrakt (möglicherweise Cascar. Sagrada und Gentiana), Natrium und Lithium in sehr geringen Mengen. Natr. bicarb., Citronensäure und Coffein, wie angegeben, sind nicht nachweisbar. - Sonnol von Dr. Schäfer's Chem. Labor. Metropol Jeipzig, Windmählenstr. 1-5, Nähr- und Kräftigungsmittel ; besteht aus etwa 30 Teilen Kakao, 65 Teilen Zucker, versetzt mit 5\% Kartoffel- oder Bananenstärke, $1 \%$ Kochsalz. - Motherils Seasick Remedy der Motheril Remedy Co. London, Mittel zur Verhütung und Auf́ebung von Seekrankheit. Preỉs $3 \mathrm{Mk}$. Schachtel mit 3 runden GelatinenSchiebekapseln, rot und braun gefärbt. Das rote Pulver besteht aus einer Mischung von Zucker, Monobromcampher und Coffein, das braune Pulver aus denselben Bestandteilen mit Citronensäure und Zimt. - Astor-Büstenpraliné der Astor-Industrie Leipzig. Preis $3 \mathrm{Mk}$. Ein weißer Karton mit 41 Stück Pralinen zu je 5-6 g; die Hülle besteht aus Kakaomasse, der gelbe Inhalt aus Nährsalzen, Zucker, Mais-, Bananen- und Leguminosenmehl, gefärbt mit Curcuma. Herniol von Dr. med. Baubolzer, München; Mittel bei akuten und chronischen Nierenleiden. Preis 2,50 Mk. Flasche mit $29 \mathrm{cem}$ einer schwarabraunen, bitteren, sehwach sauren Flüssigkeit, wahrscheinlich ein schwach alkoholischer Auszug der von alters her als Heilmittel bei Blasen- und Nierenleiden verwendeten Droge Herniaria glabra. - Tabbert'sches Wundrosenspecificum vom Chemiker Franz Tabbert, Greifswald. Mittel gegen Wundrose. Preis $5,50 \mathrm{Mk}$. Flasehe mit $253 \mathrm{ccm}$ einer nach Kamillen riechenden, süßschmeckenden Flüssigkeit, die nach kurzer Zeit in Gärung überging, bestand aus einer mit Zucker gesüßten Abkochung eines Tees. - Antisepticum Gloria von Auguste Liebig, Leipzig-Neustadt; , allen Frauen sehr empfehlenswert". Preis 1,50 Mk. Flasche mit $92 \mathrm{ccm}$ einer wasserhellen Flüssigkeit, die sich als mit Wasser verdünnte Formalinlösung darstellt. - Marubin Teint-Paste III von Otto Reichel, Berlin SO., wirksames Spezialmittel gegen Gesichtsröte, rote Nasen, rote Hände, Frost und Blutröte. Preis 2,50 Mk. Salbentopf mit $20 \mathrm{~g}$ einer dunkelbraunen, zähflüssigen Salbe, bergestellt aus einer Verreibung von Wollfett, Wasser, Ichthyol und Zinkoxyd. - Neosulfon der Chem. Industrie-Paulinum Berlin SW. 48, „wohlriechendes Schwefelbad in fester Form; mit bestem Erfolg bei inneren Krankheiten, Haut-, Geschlechts- und Frauenkrankheiten“. Preis 1,50 Mk. Präparatenglas mit $12,8 \mathrm{~g}$ einer bröckligen, gelbgraugrünen Masse, die an der Luft rasch fencht wird, 
bestehend bauptsächlich aus Alkalipolysulfiden mit einem ozonisierenden Duftträger, wahrscheinlich Terpineol. - Schweißbalsam "Probat" von E. W. Paul Koch, Halle a. S., gegen Schweißsschäden. Preis 75 Pfg. 2 Holzschachteln $72 \mathrm{~g}$ brutto Inhalt, eine sebr schlecht gemischte Salbe aus Schweinefett mil 25\% eines Zusatzes aus grob gepulvertem Alaun und einem gerbsäurebaltigen Pflanzenstoff, wahrscheinlich Catechu. - Schnupfe mit Sauerstoff von Mediz. Verlag "Medico“, Friedenau, „selbst bei chronischem Schnupfen frappierende Wirkung". Preis 7,50 Mk. Ein weißes aromatisch riechendes Pulver, bestehend aus Natriumperborat mit Rhizom. Irid. Nährabil vom Zwickauer Chem. Labor. Klaus, Zwickau i. S., Nähr- und Kräftigungsmittel bei Nervenerkrankungen, Schwächezuständen usw. Preis Mk. 3. Schachtel mit $495 \mathrm{~g}$ eines schokoladeähnlichen Pulvers, bestehend neben geringen Mengen Lecithin und Nährsalzen aus etwa 50 Teilen Erbsenmehl, 20 Teilen Kakao und 30 Teilen Zucker. Herstellungswert rund 50 Pfg. - Coelina, Menstruationstropfen vom Versandhaus Coelina, Köln a. Rh., garantiert reines Destillat aus heilkräftigen Drogen. Preis $4 \mathrm{Mk}$. Flasche mit $2 \tilde{\mathrm{o}} \mathrm{ccm}$ eines alkoholischen Destillates pflanzlicher Stoffe (angebl. Cort. cinnam., Cort. Citr., Caryoph., Herba melissae, Sem. myrist.). - Cremiona der Hirsch-Apotheke von Artur Weill, Straßburg i. E. Mittel gegen Nasenröte usw., besitzt kühlende Wirkung. Preis $3 \mathrm{Mk}$. Eine Zinntube mit $46 \mathrm{~g}$ einer gelblichweißen nach Citronenöl riechenden Salbe, hergestellt aus einer Verreibung von Mineralfett, Wasser und Aluminiumacetat, parfümiert. - Gallen-Tee von Frau A. Hencke, Erfurt. Mittel gegen Gallensteine, Gries- und Leberleiden, löst die Steine schmerzlos auf, macht Operation überflüssig. Preis Mk. 2. Pappschachtel mit $125 \mathrm{~g}$ einer Teemischung aus: Cort. Frang,, Flor. Millefol., Herb. Equiseti, Rad. Gent,, Rhizom. Rhei. - Peruyd-Fubbadpulver von Hans Schwarzkopf, G. m. b. H., Berlin. Präparat zur Pflege, Stärkung, Reinigung, Gesunderhaltung der Füße. Preis 25 Pfg. 2 Beutelchen mit je $25 \mathrm{~g}$ eines gelblichweißen parfümierten Pulvers, eines Gemisches aus Seife, Stärke, Soda, Kochsalz und einem antiseptisch wirkenden formalinhaltigen Stoff (angeblich dem nach D.R.P. hergestellten Perubalsam-Formaldehyd). - Zur Heilung von Tierkrankheiten dienten folgende zur Untersuchung eingelieferte Präparate: Rapidol-Kalkbeinsalbe von Müller und Thiede, Hamburg; Preis 1,50 Mk. Blechschachtel mit $75 \mathrm{~g}$ einer gelbgrünen Salbe, bestehend aus 75 Teilen Wollfett und 25 Teilen Formalin. - Trabin von Apotheker Gronwald \& Co., Berlin SW., gegen "Pflastermüde, Lahmbeiten, krumme Beine, steife Fesseln usw." Flasehe mit $950 \mathrm{ccm}$ einer gelben, trüben, öligen Flüssigkeit, bestehend aus einem Fischtran mit 1,5\% Zusatz eines ätherischen Öles, dem Geruche nach Eukalyptus. - Panaricin von G. Riesen, Gr. Rogahn b. Schwerin i. M., gegen Panaritium der Kübe. Preis $3,50 \mathrm{Mk}$. Flasche mit $500 \mathrm{ccm}$ einer wasserhellen Flüssigkeit, auf der $5 \mathrm{ccm}$ eines goldgelben Öles (Oleum Spicae) schwimmen; das Panarizin selbst ist eine Auflösung von 15 Teilen Zinksulfat in 100 Teilen Wasser unter Zusatz von $10 \%$ offizineller Salpetersäure. - Asthma-Pulver für Pferde von Rob. Klemm, Berlin, Mittel gegen Dämpfigkeit, Husten, Rohren, Pfeifen, Engebrust, Hartschnaufen. Preis 6 Mk. 58 weiße Pulverkapseln, bestehend aus einem Gemisch von Kochsalz, Natrium bicarb., Grauspießglanz, Kohle und Pflanzenpulver, darin Bockshornklee nachweisbar. - Herpedol vom Frisia-Laborat. Berlin-Friedenau, gegen Tellerflechte der Rinder und Pferde. Preis 2,50 Mk. Flasche mit $250 \mathrm{ccm}$ einer aus zwei Schichten bestehenden Flüssigkeit; die obere, ölige Schicht ist Terpentinöl mit etwas Fett, die untere, wässerige Schicht eine Auflösung von Ammoniak und Kresol in Wasser; das Ganze eine linimentartige Einreibung.

C. Mai.

Armin Röhrig: Kosmetische Mittel. (Jahresbericht der ehemischen Untersuchungsanstalt Leipzig 1914, 48.) -- Reinboth's Trinmph-Haarwasser, aus zwei getrennten Flüssigkeiten bestehend, von denen die obere eine Auflösung eines Pflanzenextraktes und von etwas Salpeter in wässerigem Alkohol, die untere Ricinusöl ist. - 
Blüten-Haarbalsam, von E. Pusmentirer, Leipzig, Preis $2 \mathrm{Mk}$. und 3,50 Mk., besteht aus $141 \mathrm{ccm}$ einer tiefgelben, kräftig aromatisch riechenden alkoholischen Flüssigkeit, nämlich einer gefärbten parfümierten alkoholischen 30 ;0-igen Glycerin-Lösung. - DotiExtrakt, Vorbeugungsmittel gegen Haarleiden, sowie zur Beförderung neuen, gesunden Haarwuchses, Preis 2,50 Mk. $86 \mathrm{ccm}$ einer goldgelben, nach Eau de Cologne, aber auch nach denaturiertem Spiritus riechende Flüssigkeit. Als wesentliche und wirksame Bestandteile wurden ermittelt Alkohol, Chinin, muriat., Salicylsäure und Pyridin. Biltz' Natur-Haarwell-Essenz, eine schwach alkoholische Boraxlösung (0,49\% Borsäure). - Biltz' Augenfener-Essenz ist gewöhnliches Rosenwasser. - Biltz' BüstenElixiex, eine mit Fruchtäther parfümierte, alkoholisch wässerige Lösung von Borax mit wenig Glycerin. - Biltz' Natur-Haarwuchs-Crême, eine Paraffinsalbe mit rund $1 \%$ Borax.

C. Mai.

H. Kreis: Bellin. (Bericht über die Lebensmittel-Kontrolle im Kanton Basel-Stadt 1914, 30.) - Das Desinfektionsmittel „Bellin (Tonerde.Präparat)“ gegen Frauenleiden ist eine wässerige Lösung von $3 \%$ Aluminiumchlorid. C. Mai.

S. Rabow: Übersicht der im Lanfe des Jahres 1914 bekannt gewordenen therapeutischen Neuheiten einschlieblich der Spezialitäten und Geheimmittel. (Chem.-Ztg. 1915, 39, 211-212, 241-242, 254-255 u. 263-265.)

\section{Literatur.}

Dr. L. Rosenthaler, a, o. Prof, an der Universitat Bern: Der Nachweis orga. nischer Verbindungen. A usgewählte Reaktionen und Verfahren. Band XIX/XX von: "Die chemische Analyse, Sammlung von Einzeldarstellongen anf dem Gebiete der chemischen, technisch-chemischen und physikalisch-chemischen Analyse", herausgegeben von Dr. B. M. M argosches, a. o. Professor an der deutschen Technischen Hochschule zu Brünn. XVIII u. 1070 S. Gr. 80. Stuttgart 1914, Verlag von Ferd. Enke. Preis geh. 34 Mk., geb. $35,20 \mathrm{Mk}$. - Mit diesem umfangreichen Bande der bekannten Sammlung des Enke'schen Verlages beabsichtigt der Verfasser eine didaktische Behandlung derjenigen organischen Verbinảungen, deren qualitativer Nachweis für den in der Praxis stehenden Analytiker erforderlich werden kann, vom analytischen Standpunkt aus, und zwar geleitet von dem Gedanken, auf diese Weise für die organische Chemie eine ähnliche Grundlage zu schaffen, wie sie für die anorganisehe qualitative Chemie die Werke von Fresenius, Treadwell u, a, bedeuten. Das Buch beginnt mit einer kurzen Einleitung, in der die allgemeinen Gesiehtspunkte dargelegt werden. Daran schliefst sich ein kurzes Kapitel: Nachweis einzelner Flemente. Es folgt dann (S. 11-934), in 30 Abschnitte gegliedert, die Besprechung der einzelnen Körperklassen: 1. Kohlenwasserstoffe, 2. Alkohole, 3. Aldehyde, 4. Ketone, 5. Kohlenhydrate, 6. Phenole, 7. Säuren, 8. Oxysäuren, Aldehydsïuren, Ketonsäuren, Säurederivate (Anhang Laktone, Anhydride, Säurehaloide, Acylperoxyde, Persäuren), 9. Äther, 10. Chinone, Oxyde, 11. Ester, 12. Halogenderivate, 13. Nitroderivate, Nitroso- und Isonitrosoverbindungen, 14. Nitrile und Isonitrile, 15. Säureamide and -imide, 16. Amine, aromatische Hydrazine, aromatische Diaza- und Azoverbindungen, 17. Sâurederivate organischer Basen, 18. Heterocyklische Basen, 19. Aminosäuren, Polypeptide, Betaine, 20. Organische Schwefelverbindungen (mit Ausnahme der Eiweifsstaffe), 21. Organische Arsenverbindungen, 22. Alkaloide, 23. Harzsäuren, 24. Gerbstoffe, Flechtensäuren, 25. Glykoside, 26. Bitterstoffe, 27. Farbstoffe, 28. Eiweißsstoffe, 29. Enzyme, (Fermente), 30. Toxalbumine. Daran schlieft sich weiterhin (S. 935-950) eine grofe Anzahl von Nachträgen, die die Ergebnisse der letzten Jahre (bis 1914) behandeln, eine Reagentienliste (S. 951-959); ferner umfangreiche Tabellen der Schmelz- (und Siede-)punkte der festen, und der Siede- (und Schmelz)punkte der flüssigen Körper, nach steigenden Temperaturgraden geordnet, und endlich ein ausfübrliches Sachregister, das bei den Einzelverbindungen zugleich Bruttoformel, Molekulargewicht und prozentische Zusammensetznng angibt. Ein ebenso ausführliches Autorenregister macht dann den Schluk. - Der Aufbau der einzelnen Kapitel ist klar und übersichtlich, wozu der kurze, prägnante Ausdruck ganz wesentlich beiträgt. Die naturgemäßs gebotene Beschränkung in der Behandlung der einzelnen Körper ist im allgemeinen recht glücklich, jedoch keineswegs ängstlich, durchgeführt. So geht Verf. beispielsweise bei den nahrungsmittelchemisch wichtigen Körpern oft recht grändlich za Werke und bringt dort 